\title{
Autoevaluación del aprendizaje en enfermería con ejercicios interactivos del programa Hot Potatoes
}

\author{
M.R. Rozas a , J. Costa ${ }^{b}$, L. Francés ${ }^{a}, H$. Viñas ${ }^{a}, A$. Paulí ${ }^{a}, C$. Martínez ${ }^{a}$
}

Introducción. Los ejercicios interactivos de autoevaluación son una herramienta muy útil para fomentar el aprendizaje autónomo del estudiante. El programa Hot Potatoes permite el diseño de actividades interactivas para la evaluación formativa, con la incorporación de un sistema de retroacción que refuerza los contenidos no asimilados cuando la respuesta no es correcta. El objetivo de estudio es averiguar la opinión de los estudiantes sobre la utilización del programa Hot Potatoes. Materiales y métodos. Estudio descriptivo transversal en la promoción 2006-2007 de la asignatura Enfermería Maternal II. Los datos se recogieron a través de un cuestionario diseñado ad hoc. Resultados. Los ejercicios mejor valorados y considerados de menor dificultad fueron los de opción múltiple; los más difíciles fueron los textos desordenados y los ejercicios de rellenar huecos. Todos los estudiantes consideraron que el método motivó su interés por la asignatura y facilitó su aprendizaje. El 71\% considera que el método le ha servido para adquirir conocimientos y ha sido muy útil como herramienta de estudio. Los aspectos que consideran más interesantes incluyen que: son un método de repaso y estudio, facilitan el aprendizaje de una forma amena y permiten conocer el nivel de conocimientos y corregir los errores durante el aprendizaje. Entre los aspectos que mejorarían destacan que incluirían más ejercicios y un número de preguntas más elevado. Conclusiones. El método ha tenido una buena aceptación y ha fomentado el aprendizaje autónomo. Consideramos que se trata de una estrategia de autoevaluación adecuada para la adaptación a los nuevos créditos europeos (ECTS).

Palabras clave. Autoevaluación. Hot Potatoes. Ejercicios interactivos. Evaluación formativa. Tecnologías de la información y la comunicación (TIC).

\section{Self-evaluation of nursing learning with Hot Potatoes interactive exercises}

Introduction. The interactive exercises of self-assessment are a very useful tool to foster the autonomous learning of the student.
The suite Hot Potatoes allows the design of interactive activities for the formative evaluation, incorporating a system of feedback that reinforce the not assimilated contents when the answer is not correct. The aim of study is to find out the students opinion about the utilization of the suite Hot Potatoes. Materials and methods. Cross-sectional descriptive study in Barcelona University students from Maternity Nursing. Data were gathered through a questionnaire designed ad hoc. Results. The exercises better appraised and the considerate of less difficulty ones were those of multiple-choice; the most difficult, the matching texts and gap-fill exercises. All students considered that the method had motivated their interest in the subject and it had facilitated their learning. 71\% considers that the method has been useful to acquiring knowledge and has been very useful as a study tool. The aspects that the students consider more interesting include that: they are a method of review and study, it facilitates the learning in a pleasant way, and it allows to know the level of knowledge and to correct the errors during the learning. Among the aspects that would improve they would include more exercises and questions. Conclusions. The method has had a good acceptance and has fostered the autonomous learning. We consider that it is a self-assessment tool suitable for the adaptation of the new European Credit Transfer System.

Key words. Formative evaluation. Hot Potatoes. Information and communication tehcnologies (ICT). Interactive testing tools. Selfassessment.

\section{Introducción}

La innovación desarrolla un papel fundamental en todos los niveles educativos y, en el ámbito universitario, está íntimamente relacionada con la enseñanza virtual; una de las formas básicas para su realización es la incorporación de sistemas de información, herramientas de comunicación y elementos multimedia [1]. La introducción de

\footnotetext{
a Departamento de Enfermería de Salud Pública, Salud Mental y Maternoinfantil. Escuela de Enfermería. Universitat de Barcelona. L'Hospitalet de Llobregat, Barcelona.

b Servicio de Ginecología. Hospital de Sabadell. Sabadell, Barcelona, España.

Correspondencia

M. Rosa Rozas.

Departamento de Enfermería de Salud

Pública, Salud Mental y Maternoinfantil. Escuela de Enfermería. Universitat de Barcelona. Campus de Bellvitge.

Pavelló de Govern,

3. a planta, desp. 221. E-08907 L'Hospitalet de Llobregat (Barcelona).

Fax +34 934024297

E-mail rrozas@ub.edu
} 
tecnologías de información y comunicación (TIC) recientes en la educación ha supuesto la aparición de contenidos y formas de comunicación nuevos y, sobre todo, métodos de enseñanza y aprendizaje innovadores, ya que internet proporciona gran cantidad de información y los alumnos pueden construir su aprendizaje de forma más autónoma [2].

Los sistemas de evaluación automática y, en concreto, los ejercicios interactivos de autoevaluación forman parte del proceso de autorregulación del aprendizaje, ya que permiten al alumno detectar sus errores y le ofrecen una retroacción para que pueda corregirlos durante el proceso. Además, son una herramienta muy útil a la hora de motivar a éste en las tareas de aprendizaje y permitir que sea él mismo el que constate si ya posee los conocimientos sobre los que el profesor le evaluará posteriormente [3].

La suite Hot Potatoes es un software gratuito para la educación utilizado en diversas universidades del mundo, creado por el equipo de HalfBaked Software del Centro para el Lenguaje de la Universidad de Victoria en Canadá. Esta herramienta permite la creación de ejercicios interactivos de autoevaluación para la publicación en la web. Está constituida por un conjunto de seis programas de autor que permiten construir actividades autoverificables y pruebas pedagógicas diversas en seis formatos de ejercicios distintos: elección múltiple, respuesta breve, organizar oraciones, relación de columnas, crucigramas y llenar huecos en un texto [4,5]. Aunque el programa genera cuestionarios convencionales no adaptativos, no responde al tradicional enfoque estático de los mismos, pues ofrece la posibilidad de añadir elementos de los cuestionarios dinámicos, como puede ser la inclusión de una adecuada retroacción en cada una de las posibles respuestas. Del mismo modo, el programa permite dar por válidas varias respuestas, así como incluir un reloj que limita el tiempo en el que se debe realizar la prueba. También confiere un cierto dinamismo que las preguntas y sus respuestas aparezcan en un orden aleatorio cada vez que se cargan, lo que evita el aprendizaje mecánico de éstas. En algunos casos también permite que el alumno recurra, según sus necesidades, a ciertas pistas que le ayuden a resolver el ejercicio que se presenta [6]. Todas estas características lo convierten en un programa muy adecuado para diseñar actividades de evaluación formativa a la medida de los objetivos de aprendizaje de los estudiantes.
En el nuevo contexto del Espacio Europeo de Educación Superior (EEES), el uso de las TIC en el proceso de evaluación de los estudiantes universitarios puede resultar de gran utilidad, no para introducir cambios conceptuales, sino como herramientas que nos permitan utilizar los recursos de tiempo y los materiales de manera más eficiente, tanto para el estudiante como para el profesor [7]. En la asignatura Enfermería Maternoinfantil II de la Diplomatura de Enfermería, nos planteamos introducir una innovación docente con el empleo del programa Hot Potatoes como herramienta educativa para la evaluación formativa. Elaboramos un material docente con diversas pruebas para la autoevaluación, cuya finalidad era que el estudiante pudiera construir su aprendizaje mediante su esfuerzo y su implicación activa en el proceso. Después de la puesta en marcha de la estrategia docente, el objetivo de nuestro trabajo fue conocer la opinión de los alumnos sobre este recurso pedagógico, así como determinar la carga de trabajo que supone para ellos la actividad propuesta. Los resultados de este trabajo nos han permitido valorar y reflexionar sobre la adecuación de esta metodología de enseñanza y aprendizaje a la nueva concepción de los créditos ECTS (European Credit Transfer System).

\section{Materiales y métodos}

Realizamos un estudio descriptivo transversal en el que incluimos a todos los alumnos matriculados en la asignatura Enfermería Maternal II de la Diplomatura de Enfermería del grupo M3.1 de la promoción 2006-2007 de la Escuela de Enfermería de la Universidad de Barcelona. La población estaba formada por un total de 82 alumnos. El único criterio de exclusión utilizado fue el deseo de no participar en el estudio.

Después de impartido cada tema y una vez realizadas todas las actividades que lo configuran, el alumno podía realizar los ejercicios de autoevaluación que se encontraban en el dossier electrónico de la asignatura para comprobar cuál era su nivel de aprendizaje, y reforzar los contenidos que no había asimilado o lo había hecho de forma errónea. Cada tema incluía ejercicios diferentes y, al finalizar cada uno de ellos, obtenía automáticamente la retroacción necesaria y la puntuación correspondiente. Por otro lado, y en un intento de 


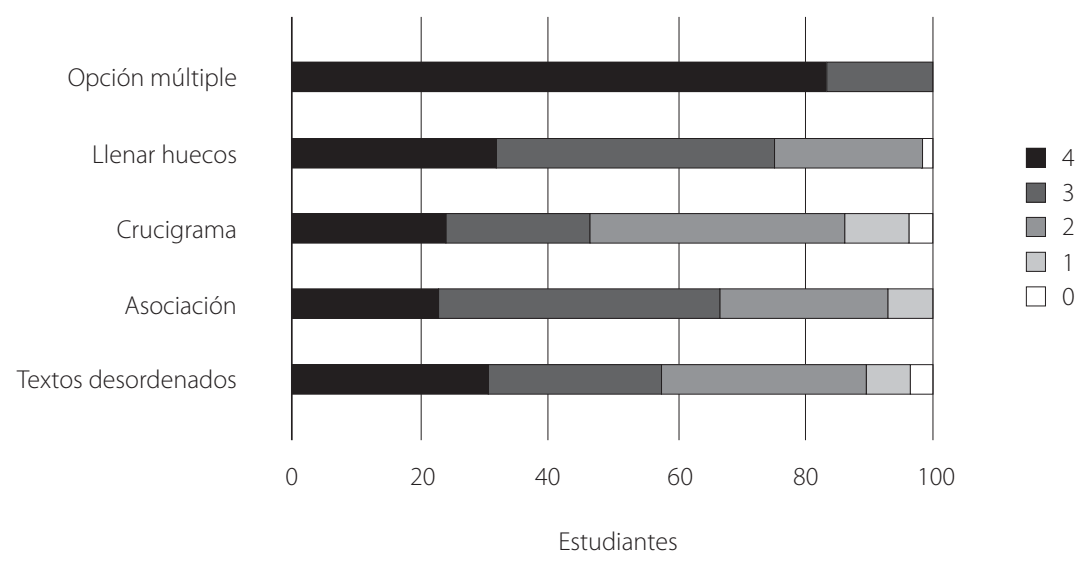

Figura 1. Utilidad de los ejercicios (escala del $0=$ poca al $4=$ mucha).

disminuir la ansiedad del alumno, al finalizar el temario incluimos dos exámenes de prueba temporizados para que se familiarizaran con el tipo de evaluación que encontrarían el día del examen. Los datos se recogieron a través de un cuestionario autocumplimentado y anónimo diseñado de forma expresa para el estudio. El cuestionario incluía las variables siguientes:

- Once variables para calificar la utilidad y la dificultad de los diferentes tipos de ejercicios propuestos: ejercicios de elección múltiple, relacionar columnas, organizar oraciones, crucigramas y llenar huecos (valorados en una escala de 0 a 4).

- Cuatro variables referentes a su aprendizaje: motivación por la asignatura, facilitación del aprendizaje y utilidad del método para adquirir conocimientos y como técnica de estudio. Estas variables se puntuaron mediante una escala de cuatro categorías (mucho, bastante, poco, nada).

- Dos variables referentes a la utilización de apuntes y libros y a la utilidad de las clases para resolver los ejercicios.

- Seis variables que consideramos que serían útiles para valorar si el método se adecuaba a la nueva concepción de los créditos ECTS: horas empleadas en la ejecución de los ejercicios, nú- mero de veces que habían realizado las pruebas, frecuencia y momento de su realización, aspectos más interesantes del método y puntos que mejorarían (encuesta abierta).

Los resultados académicos de esta promoción se compararon con los de la promoción anterior. El análisis de los datos se realizó con el paquete estadístico SPSS 12.0.1.

\section{Resultados}

De los 82 alumnos matriculados en la asignatura se recogieron 62 cuestionarios (75,6\%). De los 20 alumnos que no participaron en el estudio, sabemos que 16 eran mujeres y cuatro hombres, lo que supone un porcentaje de no participación masculina del $40 \%$, ya que sólo se incluían 10 hombres en la muestra. Del total de alumnos excluidos, 13 no entregaron el cuestionario por olvido o deseo de no participar, y siete no fueron localizados por no asistir a clase ni presentarse a ninguna de las dos convocatorias de examen.

\section{Análisis de las variables}

- Edad: la edad media de participación fue de 23,03 años (DE 6,63), con un intervalo de 19-45 años. 


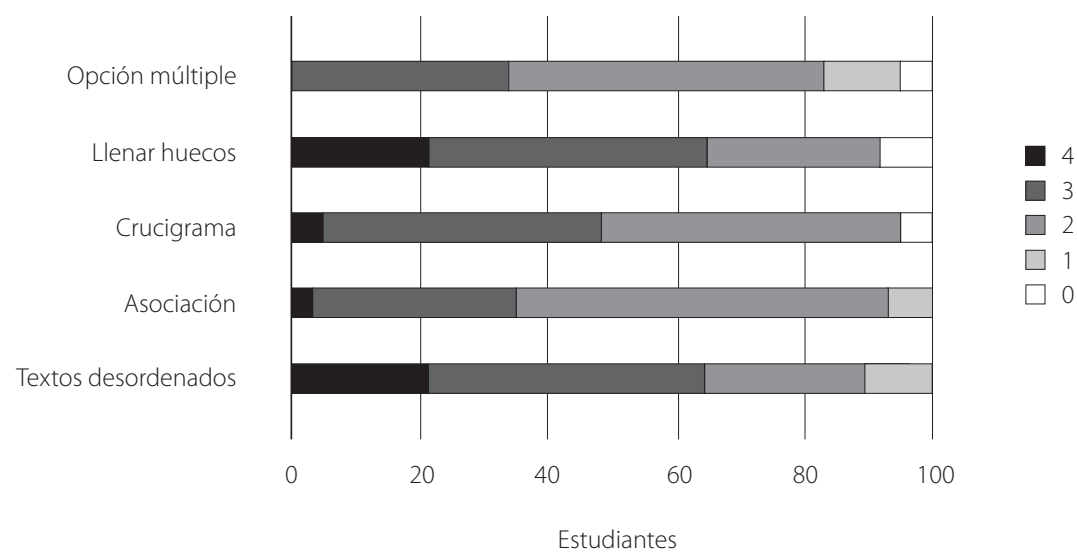

Figura 2. Dificultad de los ejercicios (escala del $0=$ poca al $4=$ mucha).

- Horas dedicadas a realizar los ejercicios: se obtuvo una media de 6,78 (DE 5,43), con un intervalo de 1-25.

- Participación en el proyecto: toda la muestra de alumnos realizó los ejercicios y más de la mi$\operatorname{tad}(56,5 \%)$ lo hizo de forma habitual; el 38,7\% lo hizo alguna vez, y el 4,8\% sólo realizó los exámenes de prueba.

- Momento de realización de los ejercicios: el 50\% los realizó en su tiempo libre, el 14,5\% después de cada tema y el 35,5\% los efectuó antes del examen.

- Número de veces que han visitado la página: el $61 \%$ lo hizo más de de 10 veces y el 38,7 , menos de 10.

- Utilidad de la asistencia a clase y de los apuntes para resolver los ejercicios: el 73,8\% indicó mucha y el $26,2 \%$, bastante. Ninguno de los participantes las calificó de poca o ninguna.

- Consulta de apuntes o libros para resolver los ejercicios: el 59,7\% indicó que en ocasiones; el $14,5 \%$ sí tuvo que consultarlos, y el $25,8 \%$ los realizó sin recurrir a la consulta.

- Utilidad de los ejercicios: en una escala del 0 al 4 , los ejercicios mejor valorados fueron los de opción múltiple, con un $83,3 \%$ de valoración 4 y un 16,7\% de valoración 3; no hubieron valores inferiores. Los ejercicios considerados menos útiles fueron los crucigramas, con pun- tuaciones de 3 y 4 en un 46,5\% de los casos (el resto de valores se muestran en la Fig. 1).

- Dificultad de los ejercicios: en una escala del 0 al 4, los ejercicios considerados de menor dificultad fueron los de opción múltiple, con puntuaciones entre 0 y 2 del 66,2\%. Los más difíciles fueron los textos desordenados y los ejercicios de llenar huecos con puntuaciones de 3 y 4 superiores al 64\% (el resto de valores se muestran en la Fig. 2).

- Utilidad de los exámenes de prueba: el 90\% de los alumnos otorgó una puntuación entre 3 y 4 , en una escala de $0-4$.

- Variables referentes al aprendizaje del alumno: en cuanto a si los ejercicios habían motivado el interés por la asignatura, los alumnos contestaron mucho $(36,1 \%)$ o bastante $(62,3 \%)$. Respecto a si los ejercicios habían facilitado su aprendizaje, el 100\% de los alumnos contestó mucho $(53,2 \%)$ o bastante $(46,8 \%)$. Todos afirmaron que este método les había servido para adquirir muchos conocimientos (71\%) o bastantes (29\%). Consideraron que esta herramienta servía como técnica de estudio, el 71\% mucho; el $25,8 \%$ bastante, y únicamente el 3,2\% poco.

Los aspectos del método que los alumnos consideraban más interesantes y los que mejorarían se muestran en las tablas I y II. 


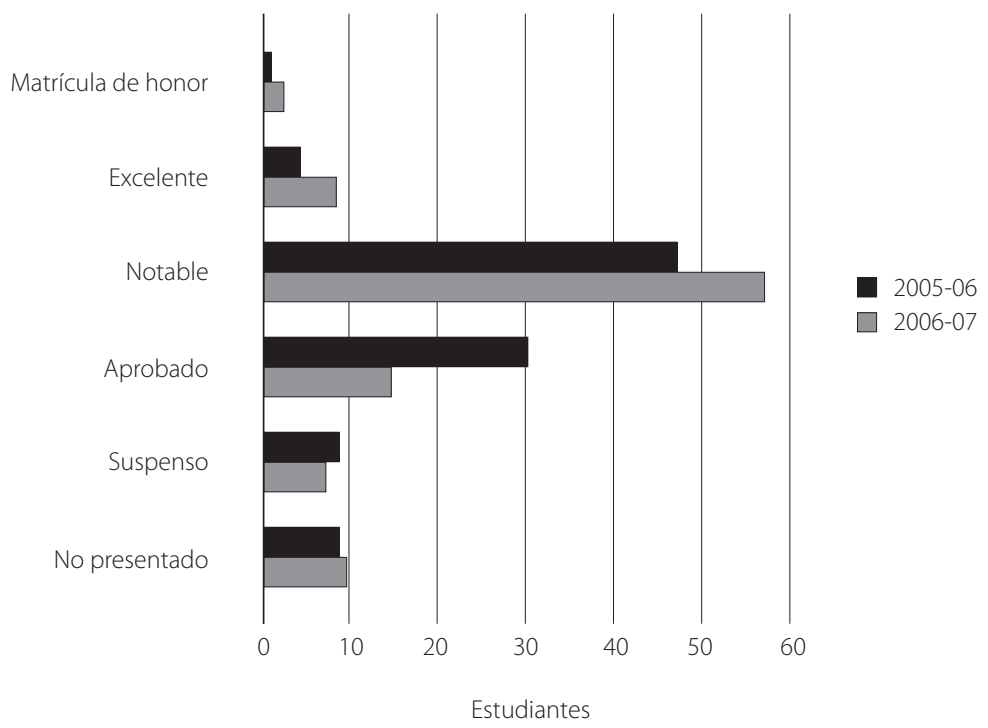

Figura 3. Comparación de los resultados académicos de la promoción de estudio (2006-2007) con los de la promoción anterior.

Se observó una mejora en los resultados académicos al compararlos con la promoción anterior, en la que no se utilizó esta metodología, de manera que aumentaron las calificaciones de excelente y notable y se obtuvo una significación estadística en los aprobados, notables y excelentes con un valor de $p=0,02$ (Fig. 3).

\section{Discusión}

A pesar del carácter voluntario de la prueba, obtuvimos un alto índice de participación. Es evidente que el aprovechamiento de estos recursos por parte de los alumnos sólo puede darse si éstos están dispuestos a acometer las nuevas responsabilidades por su propio aprendizaje, si se cuenta con docentes debidamente motivados y capacitados y, por supuesto, si existe la disponibilidad y el acceso de ambos a los recursos organizativos y tecnológicos que requiere la enseñanza virtual [8].

El hecho de que los alumnos valoraran de forma positiva la asistencia a clase, el uso de los apuntes o la consulta de libros para resolver los ejercicios nos permite constatar que la tecnología aplicada a la educación en los estudios universitarios constituye un complemento a la docencia tradicional que configura un medio de aprendizaje más dinámico, pero que no debe sustituirla [1]. Además, las actividades de autoevaluación a través de internet son insuficientes sin el apoyo de un profesorado que oriente y adapte el aprendizaje a las necesidades de los estudiantes, adaptación que se facilitará mediante la inclusión de otras actividades realizadas en el aula [9].

Los alumnos dedicaron un tiempo variable para ejecutar los ejercicios y un porcentaje elevado los realizó de forma habitual en función de su disponibilidad y de sus necesidades. Al igual que en otros estudios, constatamos que el aprendizaje autogestionado por el alumno y dirigido por el profesor (trabajo autónomo) permite una flexibilidad elevada en los tiempos y espacios dedicados al aprendizaje, y les permite compaginar el estudio con otras actividades, como por ejemplo la actividad laboral [10,11].

La mayor motivación de los alumnos que observamos con respecto al interés por la asignatura coincide con los resultados de otros estudios, 
Tabla I. Aspectos más interesantes del método.

\begin{tabular}{lcc}
\hline & $n$ & $\%^{\mathrm{a}}$ \\
\hline Es un método de repaso y estudio/guía de estudio & 14 & 22,6 \\
\hline Aprendes de forma entretenida, amena y fácil & 11 & 17,8 \\
\hline La retroacción que ofrecen los ejercicios & 10 & 16,1 \\
\hline Es un método organizado y bueno para aprender & 9 & 14,5 \\
\hline Los ejercicios de opción múltiple & 8 & 12,9 \\
\hline Permite conocer tu nivel/motiva el hacerlos bien & 6 & 9,7 \\
\hline El examen de prueba para conocer el tipo de preguntas & 6 & 9,7 \\
\hline No contestan & 15 & 24,2 \\
\hline
\end{tabular}

a La suma de porcentajes es superior a 100 porque algunos alumnos incluyeron más de un comentario.

ya que los ejercicios propuestos son elementos que captan la atención del alumnado y motivan su participación $[7,12]$. Es indudable que al profesor le supone un trabajo adicional a la hora de establecer ejercicios de este tipo, pero por regla general tienen una aceptación muy buena por parte del estudiante, que en ocasiones espera ver la creatividad del docente [1].

Las valoraciones elevadas de los alumnos respecto a si los ejercicios habían facilitado su aprendizaje, si el método les había servido para adquirir conocimientos y si constituía un método útil como técnica de estudio coinciden con las aportaciones de otros trabajos, que indican que el uso de pruebas de autoverificación, si están bien pensadas y diseñadas, pueden contribuir a orientar el proceso de aprendizaje de los alumnos y ayudarles a comprobar si lo que están aprendiendo responde efectivamente a lo que se espera de ellos [13-16].

Los estudiantes también valoraron positivamente que el aprendizaje se realizó de forma entretenida, amena y fácil debido a la gran variedad de ejercicios, aunque los considerados más fáciles y más útiles fueron los cuestionarios de opción múltiple. Cualquier modelo de autoevaluación debe incluir o permitir variedad de recursos (gráficas, imágenes, etc.) [17], ya que el objetivo es mantener o incrementar la curiosidad y el interés del alumno por la asignatura, y generar documentos dinámicos que disminuyan la fatiga que representa tener que acceder siempre al mismo contenido y de la misma forma [18]. Al igual que el profesor, los estudiantes se encuentran inmersos en la sociedad de la información y la integración de las TIC en la docencia universitaria resulta un atractivo sobre el sistema de docencia clásico [1]. Coincidimos con otros autores en que el modo de presentación de la evaluación puede influir significativamente en el rendimiento de los estudiantes y que un diseño apropiado de las pantallas es un factor importante en la evaluación en línea [19]. No obstante, no hemos de olvidar que cualquier innovación basada en las TIC no debe ser sólo un recurso metodológico sino que ha de tener una finalidad educativa. La innovación debe proporcionar un avance pedagógico y no únicamente tecnológico, sin olvidar que el sujeto principal es el estudiante y el proceso educativo [20,21].

La retroacción incluida en los diferentes ejercicios ha demostrado ser un elemento motivador 
Tabla II. Aspectos que mejorarían el método.

\begin{tabular}{|c|c|c|}
\hline & $n$ & $\%^{\mathrm{a}}$ \\
\hline Ninguno & 14 & 22,6 \\
\hline Retroacción en todos los ejercicios & 13 & 21 \\
\hline Mayor número de preguntas y de ejercicios & 9 & 14,5 \\
\hline Menos crucigramas y ejercicios de asociación & 3 & 4,8 \\
\hline No contabilizar las faltas ortográficas & 2 & 3,2 \\
\hline Incluir casos clínicos & 2 & 3,2 \\
\hline Mayor dificultad de los ejercicios & 1 & 1,6 \\
\hline No temporizar los exámenes de prueba & 1 & 1,6 \\
\hline Aplicar el método a otras asignaturas & 1 & 1,6 \\
\hline No contestan & 18 & 29 \\
\hline
\end{tabular}

a La suma de porcentajes es superior a 100 porque algunos alumnos incluyeron más de un comentario.

del aprendizaje para los alumnos. En nuestro trabajo, al igual que en otros estudios que analizan el rendimiento de los alumnos en experiencias de evaluación formativa, los elementos más valorados son: la rapidez de la respuesta y la retroacción que en ella se ofrece $[16,19,22]$. Al estudiante le interesa que exista la retroacción, ya que enlaza su producción con los resultados, se le ofrecen ayudas y orientaciones, y todo ello produce una motivación para seguir con sus estudios [11]. Hay que destacar que lo importante es la calidad de la retroacción que estas herramientas nos permiten tener. Es decir, utilizar la evaluación en su modalidad formativa en la que se ofrezca al estudiante información detallada de su actuación [7,23].

Los estudiantes opinaron que este tipo de ejercicios permite conocer su nivel y también valoraron positivamente los exámenes de prueba incluidos en el paquete de ejercicios. La retroacción que se ofrece al alumno al interaccionar con el programa permite incrementar la seguridad con la que afrontará posteriormente un examen, sabiendo de antemano cuál es el grado de profun- didad y dominio del conocimiento del que se le va a examinar. De esta forma, el alumno reduce la ansiedad y el nerviosismo con que realiza la prueba final, y mejora la nota media [18]. Para constatar este hecho y como mecanismo de evaluación interna de la mejora de la docencia [17] realizamos un análisis de los resultados del aprendizaje de los estudiantes y lo comparamos con los resultados obtenidos en la promoción académica anterior. El aumento de las calificaciones de excelente, notable y aprobado sobre los suspensos y no presentados coincide con los resultados obtenidos en otros estudios [18]. No obstante, no podemos afirmar definitivamente que la mejora de los resultados observados sea debida a los ejercicios de autoevaluación propuestos, aunque el análisis exploratorio de datos procedentes de diversas experiencias hace pensar que es verosímil suponer que tal efecto existe [24].

Al igual que en otros estudios, los participantes manifestaron más aspectos positivos que negativos al utilizar esta metodología de aprendizaje. Incluso los aspectos que los estudiantes mejo- 
rarían del método nos permiten afirmar que la herramienta ha tenido una buena aceptación por parte de éstos (Tablas I y II) [16,25].

No se ha evaluado la carga de trabajo que esta innovación ha supuesto para el docente y que podría ser objeto de un estudio posterior. Es obvio que el material pedagógico elaborado expresamente para el uso mediante las nuevas tecnologías necesita aportar un valor añadido, y esto supone un incremento del tiempo de preparación y dedicación del profesor. También implica que el docente tiene que desempeñar unos roles a los que en ocasiones no estaba acostumbrado, como desarrollar habilidades con las TIC, lo que supone una mayor dedicación de tiempo $[1,10]$.

En conclusión, el estudio realizado ha reflejado que los alumnos han sido conscientes de los beneficios de la utilización del programa Hot Potatoes y del atractivo de esta metodología. Como conclusiones podemos afirmar que la autoevaluación:

- Tiene una buena aceptación por parte de los estudiantes.

- Fomenta el aprendizaje autónomo, que implica al alumno en el proceso.

- Favorece la evaluación formativa (retroacción).

- Nos permite contabilizar la dedicación del estudiante.

- Los resultados académicos han sido superiores a los de la promoción anterior.

- La inclusión de herramientas de evaluación mediante TIC puede ayudar al estudiante a construir su aprendizaje.

- Consideramos que constituye un método adecuado para la nueva concepción de los créditos ECTS.

La implantación de esta metodología de enseñanza y aprendizaje a través de internet ha permitido que los estudiantes hayan disfrutado de una variante metodológica sin las restricciones de tiempo y espacio, y así aprender a un buen ritmo, sin requerir una excesiva dedicación ni que resultara una carga. El estudiante ha autogestionado su tiempo y profundizado en su aprendizaje según sus necesidades y ha tenido una mayor autonomía e implicación en su proceso de aprendizaje. Es importante para los docentes que vivimos estos momentos de cambios en el proceso educativo, donde las TIC adquieren cada vez más protagonismo en los procesos de enseñanza-aprendizaje, prestar atención a los instrumentos que surgen y que pueden facilitar el trabajo con la tecnología, como el mencionado en este trabajo. Además, los resultados de este estudio nos permiten reflexionar sobre la utilidad de estas estrategias docentes y su adaptación a los objetivos de aprendizaje de los estudiantes.

\section{Bibliografía}

1. Carabantes D, Carrasco A, Alves JD. La innovación a través de entornos virtuales de enseñanza y aprendizaje. Revista Iberoamericana de Educación a Distancia (RIED) 2005; 8: 105-26.

2. Mur F. El uso de la web docente en el aula. Ecomur.com. URL: http://w3.cnice.mec.es/cinternet-educacion/2congreso_actas/documentos/experiencias/pdf/foro1/ Fernando_Mur_Alegre_EL_uso_de_la_web_docente_ en_el_aula.pdf. [17.09.2007].

3. Pastor C. Herramientas de autoevaluación tipo test online y off-line freeware: Hot Potatoes y SED URL: http:// www.virtualeduca.org/virtualeduca/virtual/actas2002/ actas02/708.pdf. [17.09.2007].

4. Arneil S, Holmes M, Street H. Hot Potatoes. Language Learning \& Technology 2001; 5: 28-33. URL: http://llt. msu.edu/vol5num2/review3/default.html. [17.09.2007].

5. Ibabe I, Jauregizar J. Ejercicios de autoevaluación con Hot Potatoes. In Ibabe I, Jauregizar J, eds. Cómo crear una web docente de calidad. A Coruña: Netbiblo; 2005.

6. Raña JC. Hot Potatoes y las ciencias sociales. A pie de aula. URL: http://www.profes.net/anteriores2.asp?anteri ores $=$ Geograf $\%$ EDa + e + Historia\&id_contenido $=32905$. [17.09.2007].

7. Rodríguez MJ. Aplicación de las TIC a la evaluación de alumnos universitarios. Revista Electrónica Teoría de la Educación 2005; 6. URL: http://www3.usal.es/ teoriaeducacion. [17.09.2007].

8. Dorrego E. Educación a distancia y evaluación del aprendizaje. Revista de Educación a Distancia (RED) 2006; M6. URL: http://www.um.es/ead/red/M6. [17.09.2007].

9. Rañá JC. Algunas consideraciones sobre las actividades de autoevaluación a través de internet. URL: http://clio.rediris.es/n30/segunda/autoevaluacion.htm. [17.09.2007].

10. Canós L, Ramón F. Aplicación de las nuevas tecnologías para el trabajo autónomo del alumno. URL: http://w3.iec. csic.es/URSI/articulos_gandia_2005/articulos/ED4/527. pdf. [17.09.2007].

11. Charman D. Issues and impacts of using Computer-Based Assessments (CBA) for formative assessment. In Race P, 
Brown S, Bull J, eds. Computer-assisted in higher education. London: Kogan Page; 1999.

12. Salinas J. Innovación docente y uso de las TIC en la enseñanza universitaria. Revista de Universidad y Sociedad del Conocimiento (RUSC) 2004; 1. URL: http://www.uoc. $\mathrm{edu} / \mathrm{rusc} / \mathrm{dt} / \mathrm{esp} /$ salinas1104.pdf. [17.09.2007].

13. Lara S. La evaluación formativa en la Universidad a través de internet: aplicaciones informáticas y experiencias prácticas. Pamplona: Ediciones de la Universidad de Navarra; 2001.

14. Lavié JM. La evaluación del aprendizaje a través de internet. URL: http://tecnologiaedu.us.es/edutec/paginas/92. html. [17.09.2007].

15. Korhonen A, Malmi L, Nikander J, Tenhunen P. Interaction and feedback in automatically assessed algorithm simulation exercises. Journal of Information Technology Education 2003; 2: 241-54. URL: http://jite.org/documents/Vol2/v2p241-255-24.pdf. [17.09.2007].

16. Guiteras J. Sistema de evaluación de contenidos de embriología utilizando la computadora. URL: http://www. forumestudiantil.sld.cu/salones-presenciales/productosterminados-i/sistema-de-evaluacion-de-contenidosde-embriologia-utilizando-la-computadora.doc?set language $=$ es. [17.09.2007].

17. Díaz MA, Martínez AB, Riesco M, Iglesias C. E-valuate: un modelo de autoevaluación para la mejora de la enseñanza y el aprendizaje. URL: http://www.di.uniovi.es/ albizu/articulos/jenui03b.pdf. [17.09.2007].

18. Antelm JM, Mollá R, Vivó R, Vidal V, Robles A, Gil ML. Sistema de autoevaluación multimedia a distancia. URL:
http://www.sig.upv.es/websig/docencia/docs/SAM\%20 CIEET.pdf. [17.09.2007].

19. Ricketts C, Wilks SJ. Improving student performance through computer-based assessment: insights from recent research. Assessment \& Evaluation in Higher Education 2002; 27: 475-9.

20. Ramiro E. Una xarxa plena de TIC (metodologia per a la classificació de pàgines web educatives i el seu aprofitament). Revista de Educación a Distancia (RED) 2004; 11. URL: http://www.um.es/ead/red/11/xarxa.pdf. [17.09.2007].

21. García-Areitio L. Formación a distancia para el nuevo milenio. ¿Cambios radicales o de procedimiento? URL: http://www.uv.es/soespe/bordon52-3.html\#Marcelo. [17.09.2007].

22. Lara S. La evaluación formativa a través de internet. In Cebrián M, ed. Enseñanza virtual para la innovación universitaria. Madrid: Narcea; 2003.

23. Taras M. To feedback or not to feedback in student selfassessment. Assessment \& Evaluation in Higher Education 2003; 28: 549-65.

24. González JA, Cobo E, Martí M, Muñoz P. Desarrollo y aplicación de nuevas tecnologías para la formación universitaria. Teoría de la educación: educación y cultura en la Sociedad de la Información 2006; 7. URL:http://www. usal.es/ teoriaeducacion/rev_numero_07/n7_art_gonzalez_ cobo_marti_munoz.htm. [17.09.2007].

25. Stephens D. Use of computer assisted assessment: benefits to students and staff. Education for Information 2001; 19: 265-75. 
